\title{
Does diacylglycerol regulate KCNQ channels?
}

\author{
Byung-Chang Suh $\cdot$ Bertil Hille
}

Received: 27 March 2006 / Accepted: 18 April 2006 / Published online: 24 May 2006

(C) Springer-Verlag 2006

\begin{abstract}
Some ion channels are regulated by inositol phospholipids and by the products of cleavage by phospholipase C (PLC). KCNQ channels (Kv7) require membrane phosphatidylinositol 4,5-bisphosphate $\left(\mathrm{PIP}_{2}\right)$ and are turned off when muscarinic receptors stimulate cleavage of $\mathrm{PIP}_{2}$ by PLC. We test whether diacylglycerols are also important in the regulation of KCNQ2/KCNQ3 channels using electrophysiology and fluorescent translocation probes as indicators for $\mathrm{PIP}_{2}$ and diacylglycerol in ts $\mathrm{A}$ cells. The cells are transfected with $\mathrm{M}_{1}$ muscarinic receptors, channel subunits, and translocation probes. Although they cause translocation of a fluorescent probe with a diacylglycerol-binding $\mathrm{C} 1$ domain, exogenously applied diacylglycerol (oleoyl-acetyl-glycerol and dioctanoyl glycerol) and phorbol ester do not mimic or occlude the suppression of KCNQ current by muscarinic agonist. Blocking the metabolism of endogenous diacylglycerol by inhibiting diacylglycerol kinase with R59022 or R59949 slows the decay of diacylglycerol twofold but does not mimic or occlude muscarinic regulation and recovery of current. Blocking diacylglycerol lipase with RHC-80267 also does not occlude muscarinic modulation of current. We conclude that the diacylglycerol produced during activation of PLC, any activation of protein kinase $\mathrm{C}$ that it may stimulate, and downstream products of its metabolism are not essential players in the acute muscarinic modulation of KCNQ channels.
\end{abstract}

B.-C. Suh $\cdot$ B. Hille $(\bowtie)$

Department of Physiology and Biophysics,

University of Washington School of Medicine,

G-424 Health Sciences Building, P.O. Box 357290 Seattle,

WA 98195-7290, USA

e-mail: hille@u.washington.edu
Keywords Diacylglycerol · Phospholipase C . Inositol 1,4,5-trisphosphate $\cdot$ Phosphatidylinositol 4,5-bisphosphate $\cdot$ Muscarinic receptor $\cdot$ M-current Protein kinase C $\cdot 59022 \cdot$ RHC-80267

\section{Introduction}

The various organellar membranes and the plasma membrane each has a unique lipid composition, and their integral and peripheral membrane proteins are sensitive to these lipids [1]. Thus, the plasma membrane contains much of the cellular phosphoinositide, phosphatidylinositol 4,5bisphosphate $\left(\mathrm{PIP}_{2}\right)$, as well as numerous $\mathrm{PIP}_{2}$-sensitive proteins [2, 3]. KCNQ ion channels (Kv7.2 and Kv7.3) underlie the classical $\mathrm{M}$ current of sympathetic neurons, a non-inactivating $\mathrm{K}^{+}$current that can be suppressed by agonists that activate phospholipase C (PLC). Recently, we and others presented evidence that KCNQ channels require $\mathrm{PIP}_{2}$ for function and that they are suppressed by PLC because $\mathrm{PIP}_{2}$ becomes depleted from the plasma membrane [4-10]. The evidence included showing that PLC is essential for agonist-induced suppression of current, that $\mathrm{PIP}_{2}$ is dramatically depleted when PLC is activated, that other molecules that bind up $\mathrm{PIP}_{2}$ also suppress current, that ATP and a lipid kinase on the PIP $_{2}$-synthesis pathway are essential for recovery of current, that recovery had a similar time course to $\mathrm{PIP}_{2}$ regeneration, and that exogenously applied short-chain $\mathrm{PIP}_{2}$ analogs can speed recovery and slow suppression. We have made a kinetic model that successfully describes the events of muscarinic activation of PLC, hydrolysis, and resynthesis of $\mathrm{PIP}_{2}$, and the resulting effects on KCNQ currents [5, 8]. 

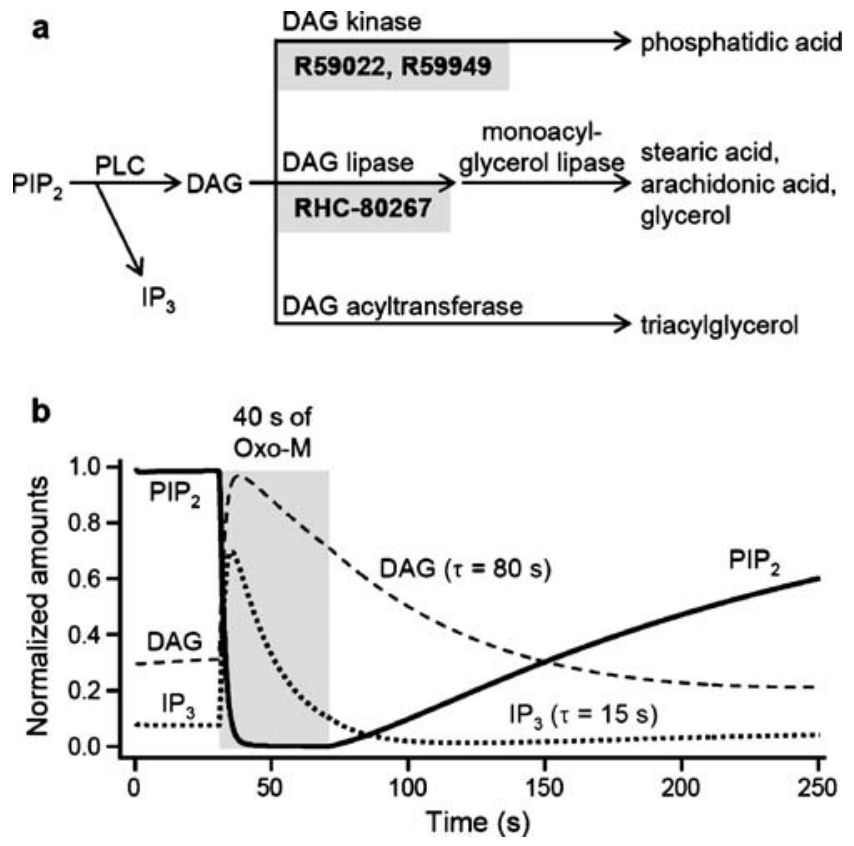

Fig. 1 a, b Products of $\mathrm{PIP}_{2}$ cleavage by phospholipase C (PLC). a Pathways of diacylglycerol (DAG) metabolism following cleavage of phosphatidylinositol 4,5-bisphosphate $\left(\mathrm{PIP}_{2}\right)$ by PLC, showing three pathways catalyzed by: DAG kinase, sequential action of DAG lipase and monoacylglycerol lipase, and DAG acyltransferase. Gray boxes indicate steps blocked by inhibitors used in our study. $\mathbf{b}$ Simulation of the time course of $\mathrm{PIP}_{2}, \mathrm{DAG}$, and inositol 1,4,5-trisphosphate $\left(\mathrm{IP}_{3}\right)$ following activation of PLC for $40 \mathrm{~s}$ by a muscarinic agonist using the kinetic model of Horowiz et al. 2005. Numbers in parentheses $(\tau)$ are the assumed mean lifetimes of DAG and $\mathrm{IP}_{3}$ in the simulation

In this paper, we continue to test the hypothesis that depletion of $\mathrm{PIP}_{2}$ suffices to explain muscarinic suppression of KCNQ currents and that resynthesis of $\mathrm{PIP}_{2}$ suffices to explain recovery of current. Activation of PLC not only depletes $\mathrm{PIP}_{2}$, but it also produces a cascade of lipidic and soluble signaling products, the first of which are diacylglycerol (DAG) and inositol 1,4,5-trisphosphate ( $\mathrm{IP}_{3}$ ) (Fig. 1a). Each of the products needs to be investigated to know if it also contributes to PLC-mediated suppression or recovery of KCNQ current.

This paper focuses on possible roles of DAG in the short time scale of acute modulation of current. Is it essential for current modulation? Do DAG or DAG metabolites have significant direct or indirect effects on the channels during acute channel modulation? There is precedent for sensitivity to DAG. Older studies reported that activation of protein kinase $\mathrm{C}$ (PKC) by DAGs and phorbol esters can reduce $\mathrm{M}$ current [11-14], and newer papers suggest that KCNQ channels exist in a complex with bound PKC and can be phosphorylated [15]. In addition, some other ion channels are thought to be directly responsive to DAG independent of PKC [16-18]. We express KCNQ channels from their constituent KCNQ2 and KCNQ3 subunits together with PLC-coupled $\mathrm{M}_{1}$ muscarinic receptors in a mammalian cell line. The experiments consist of studying effects of adding exogenous DAG analogs and of slowing the metabolism of endogenous DAG. We use two fluorescent translocation probes (1) for DAG to verify its appearance in the plasma membrane and (2) for $\mathrm{PIP}_{2}$ to verify its depletion during activation of receptors.

\section{Materials and methods}

Human embryonic kidney tsA-201 (tsA) cells were cultured and transiently transfected using Lipofectamine 2000 (Invitrogen, Carlsbad, CA, USA) with various cDNAs, as described in [8]: $\mathrm{M}_{1}$ muscarinic receptor $(1 \mu \mathrm{g}$, from Neil Nathanson, University of Washington, USA), PH-PLC $81-$ EGFP (PH-EGFP, $0.25 \mu \mathrm{g}$, from Pietro De Camilli, HHMI, Yale University, USA), PKC-C1A-EGFP (C1-EGFP, $0.25 \mu \mathrm{g}$, from Tobias Meyer, Stanford University), the channel subunits human KCNQ2 and rat KCNQ3 (Kv7.2 and $\mathrm{Kv} 7.3 ; 1 \mu \mathrm{g}$, from David McKinnon, State University of New York, Stony Brook, NY, USA), and green fluorescent protein $(0.1 \mu \mathrm{g})$ as a marker for transfection if needed. The muscarinic receptor agonist oxotremorine-M was used at $10 \mu \mathrm{M}$. Chemicals were purchased from Sigma-Aldrich (St. Louis, MO, USA).

For translocation experiments, tsA cells were imaged 24-48 h after transfection [8]. Images were taken every $5 \mathrm{~s}$ on a Leica TCS/MP confocal microscope at room temperature and analyzed, as described [5]. In the legends, we use the symbol $F$ to represent the mean pixel intensity from the fluorescent probe in a cytoplasmic (in one case nuclear) region of interest, normalized so that the minima and maxima are 0 and 1.0. For electrophysiology experiments, cells were locally perfused with flowing solutions as KCNQ currents were recorded by whole-cell patch clamp, as described in [7]. Currents were studied by holding the cell at $-20 \mathrm{mV}$ and applying a $500 \mathrm{~ms}$ hyperpolarizing step to $-60 \mathrm{mV}$ every $4 \mathrm{~s}$. The plotted amplitude of the current is the outward current at the $-20 \mathrm{mV}$ holding potential. The voltage dependence of channel activation was measured from the normalized amplitudes of outward currents at $-70 \mathrm{mV}$ (tail currents) after 500-ms depolarizations to various potentials. The plotted value is the mean current in the period $10-20 \mathrm{~ms}$ after return to $-70 \mathrm{mV}$.

Kinetic simulations are made with the virtual cell environment of the National Resource for Cell Analysis and Modeling, University of Connecticut Health Center http://www.nrcam.uchc.edu. We started with the model described in Horowitz et al. 2005 [5] and made small changes. The revised working model with control values of rate constants and initial conditions is available at that web page for public use and modification under Shared/hillelab/ SuhHilleEJPFig $1 b$. 
Fig. 2 a-f Oleoyl-acetyl-glycerol (OAG) and dioctanoyl glycerol (DOG) translocate the C1-EGFP probe to the plasma membrane. Confocal images of C1-EGFP (a) and PH-EGFP

(b) probes transiently expressed in tsA cells, are shown in negative contrast. Cells were bathed with $10 \mu \mathrm{M}$ of oxotremorine-M (Oxo-M) or, where indicated by bars above image, with OAG. Calibration bars, $10 \mu \mathrm{m}$.

c Representative time course (5-s sample intervals) of mean fluorescence per pixel in a cytoplasmic (open circles) and a nuclear region-of-interest (filled circles) of a cell expressing the C1-EGFP probe. Oxo-M and OAG were applied where indicated. d Cytoplasmic and nuclear fluorescence in a similar experiment, but with the $\mathrm{PH}-$ EGFP probe. e, f Representative time courses of cytoplasmic and a nuclear fluorescence for $\mathrm{C} 1$ EGFP (e) and PH-EGFP (f) during application of $10 \mu \mathrm{M}$ DOG instead of OAG

a

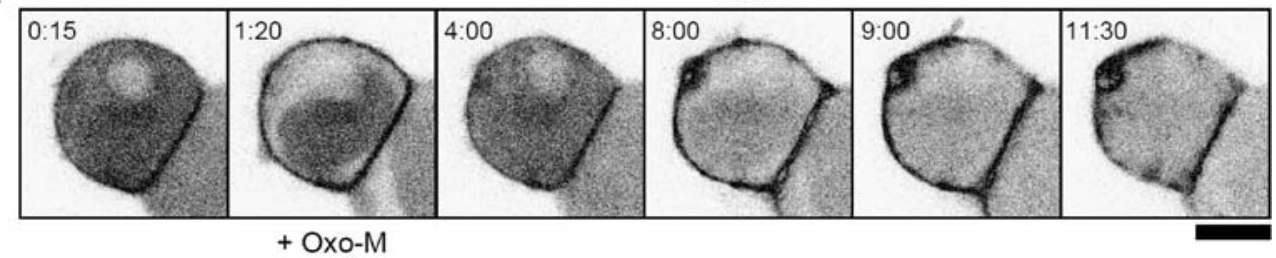

b

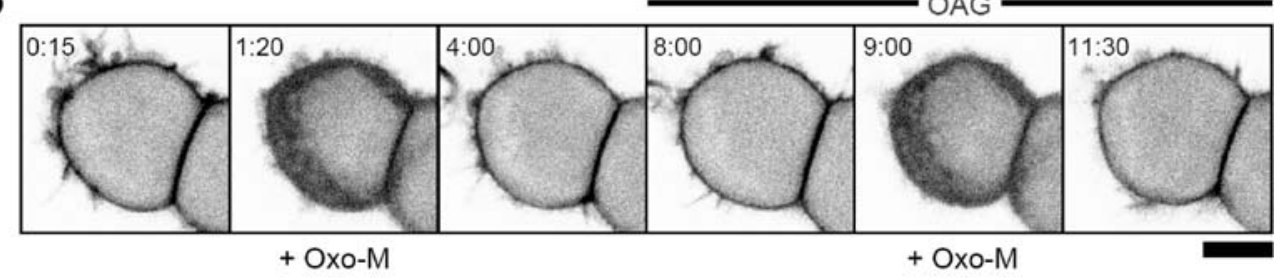

C

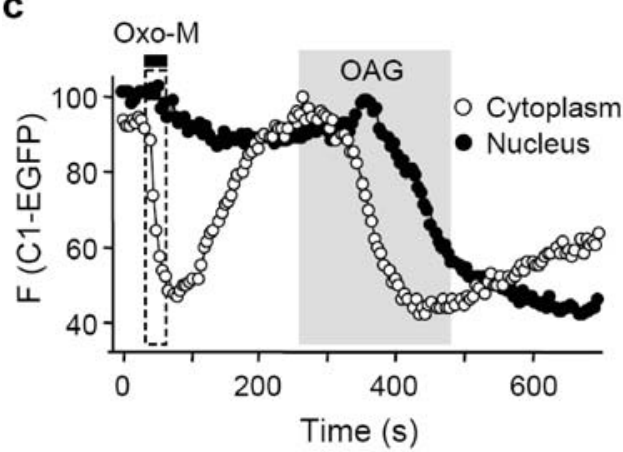

e

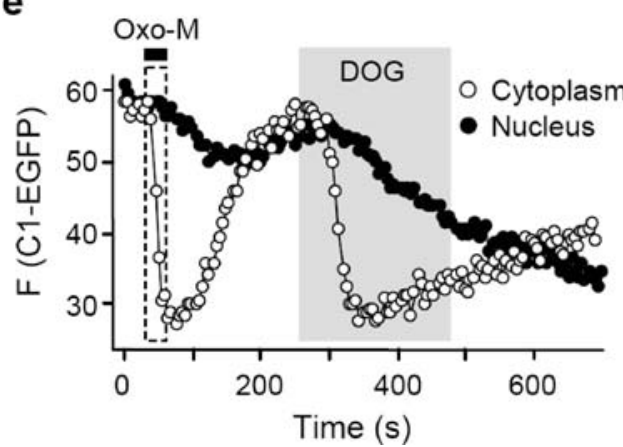

d

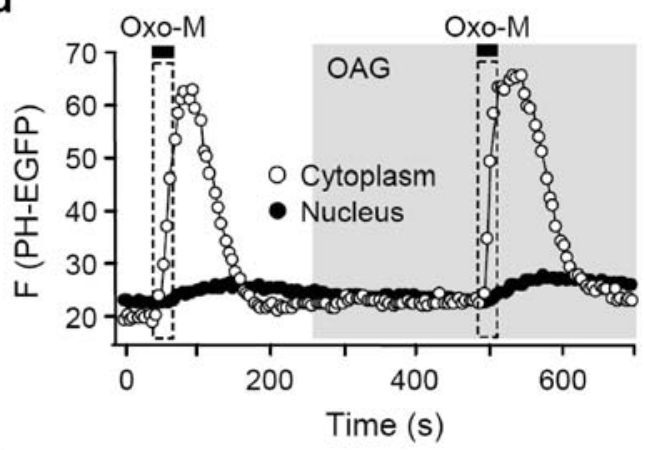

f

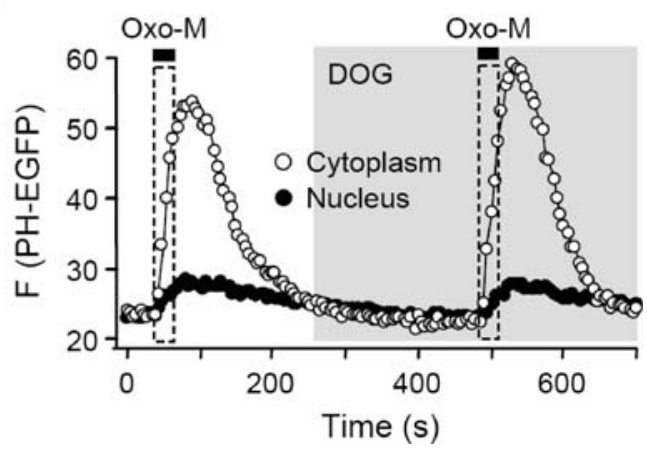

\section{Results}

Modeling of DAG and $\mathrm{IP}_{3}$ kinetics

We begin by showing the time course of receptor-evoked production and disappearance of $\mathrm{DAG}$ and $\mathrm{IP}_{3}$ predicted by our previous kinetic modeling (Fig. 1b). As in our previous presentations of this model [5,8], application of a supramaximal concentration $(10 \mu \mathrm{M})$ of the muscarinic agonist oxotremorine-M (Oxo-M) activates PLC and causes $\mathrm{PIP}_{2}$ to fall 100-fold within a few seconds. Recovery of $\mathrm{PIP}_{2}$ begins only after the agonist is removed. All of the $\mathrm{PIP}_{2}$ that is cleaved is converted into equimolar amounts of DAG and $\mathrm{IP}_{3}$. The time course of these two products would be identical if they had the same lifetime; however, it seems that they are further metabolized at different rates. The model assigns DAG molecules a mean lifetime of $80 \mathrm{~s}$, chosen to match our experiments with translocation of the C1-EGFP probe for DAG [5] (see also below). This decay of DAG represents the parallel actions of the three enzymes shown in Fig. 1a. The model assigns $\mathrm{IP}_{3}$ molecules a mean lifetime of $15 \mathrm{~s}$, representing rapid dephosphorylation by $\mathrm{IP}_{3}$ 5-phosphatase.

The literature does not have accurate measures of $\mathrm{IP}_{3}$ lifetime, but our value would allow $\mathrm{IP}_{3}$-dependent calcium release to fall rapidly after agonist is removed, as in our published experiments [5]. An interesting point to note is that because $\mathrm{PIP}_{2}$ is quickly depleted, the rate of production of $\mathrm{IP}_{3}$ and DAG must fall dramatically while the muscarinic agonist is still present. This is particularly evident in the time course of short-lived $\mathrm{IP}_{3}$. In functional experiments that study $\mathrm{IP}_{3}$-induced calcium release, the potential 
Fig. 3 a-f Effects of DAG analogs on KCNQ current in tsA cells. a, d DAG analogs and muscarinic modulation of current. Oxo-M $(10 \mu \mathrm{M})$ was applied 5 min after the addition of $10 \mu \mathrm{M}$ of OAG (a) or DOG (d). b, e Aligned current waveforms before and after $5 \mathrm{~min}$ of OAG (b) or DOG (e) application. Dashed line in the current traces is the zero-current level. c, $\mathbf{f}$ Voltage-dependence of tail-currents at $-70 \mathrm{mV}$ in control and after OAG or DOG treatment
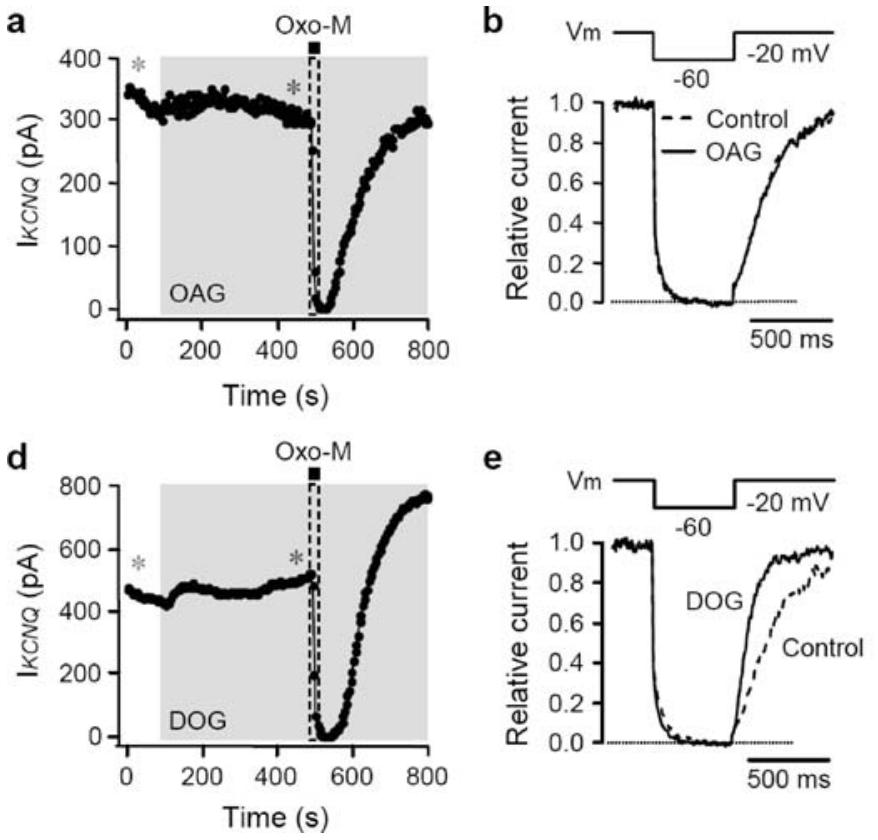

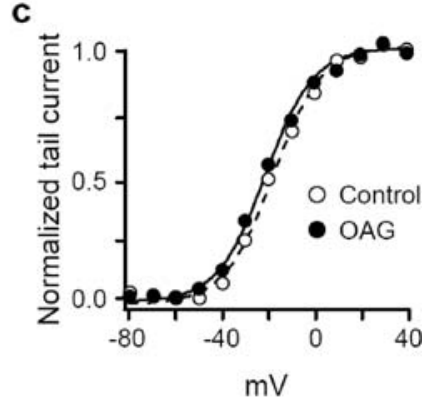

f

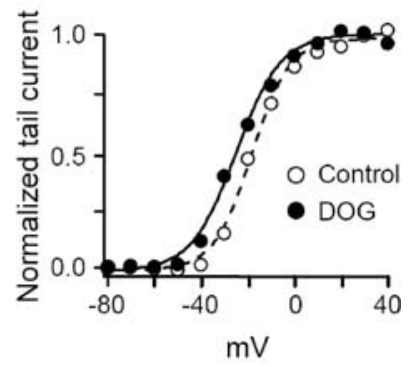

depletion of $\mathrm{PIP}_{2}$ is often overlooked as a possible explanation for transients in $\mathrm{IP}_{3}$ production and in calcium elevations. In some systems, the depletion of PIP 2 by PLC is probably much less severe than in this model, as there is a concomitant activation of the lipid kinases that synthesize $\mathrm{PIP}_{2}$. The factors regulating these lipid kinases are not wellunderstood.

\section{Application of DAGs}

The first experiments studied actions of two exogenously applied synthetic DAGs, 1-oleoyl-2-acetyl-sn-glycerol (OAG) and 1,2-dioctanoyl-sn-glycerol (DOG). Figure 2 shows confocal control experiments monitoring the translocation of a fluorescent probe for DAG, C1-EGFP [19, 20], and of a fluorescent probe that binds both to $\mathrm{PIP}_{2}$ and $\mathrm{IP}_{3}$, PH-EGFP [21]. The cells are transfected with one of these probes and with $M_{1}$ muscarinic receptors to permit activation of PLC by a muscarinic agonist.

In the resting condition, the DAG probe appears throughout the cytoplasm and nucleus, as there is little membrane DAG for it to bind to (Fig. 2a, first image), and the $\mathrm{PIP}_{2} / \mathrm{IP}_{3}$ probe is bound to the plasma membrane where there is much $\mathrm{PIP}_{2}$ (Fig. 2b, first image). Then Oxo-M is applied to activate PLC. The DAG probe translocates from cytoplasm to plasma membrane as DAG is formed there, and the $\mathrm{PIP}_{2} / \mathrm{IP}_{3}$ probe migrates the other way as membrane $\mathrm{PIP}_{2}$ is cleaved and cytoplasmic $\mathrm{IP}_{3}$ is formed (second images of Fig. 2a,b). The effect is reversible after Oxo-M is removed (third images), showing that DAG is metabolized, $\mathrm{PIP}_{2}$ is resynthesized, and presumably $\mathrm{IP}_{3}$ is broken down, all within $<200 \mathrm{~s}$.
The full-time courses of the transient changes of cytoplasmic probe fluorescence for this experiment are drawn in Fig. 2c,d as open symbols. Fluorescence in the nucleus is shown as filled symbols. At about $250 \mathrm{~s}, 10 \mu \mathrm{M}$ $\mathrm{OAG}$ is applied. It induces slow migration of the DAG probe to the plasma membrane (Fig. 2a,c), showing that enough applied OAG penetrates gradually to the inner leaflet of that membrane to attract the C1-domain of PKC. This prolonged recruitment lasts long enough to draw probe from the nucleus as well (see [5]). Nevertheless, OAG does no affect the localization of the $\mathrm{PIP}_{2} / \mathrm{IP}_{3}$ probe or its subsequent reversible translocation during application of Oxo-M (Fig. 2b,d), showing that PLC can be activated, the products metabolized, and new $\mathrm{PIP}_{2}$ can be synthesized in normal fashion in the presence of added OAG. Figure 2e,f shows similar experiments with $10 \mu \mathrm{M}$ DOG instead of OAG. The results look virtually the same $(n=5)$.

Having shown that $10 \mu \mathrm{M}$ OAG or DOG suffice for translocation of the DAG probe, our next experiment tested if these exogenous DAGs affect expressed KCNQ channels and their modulation. OAG $(10 \mu \mathrm{M})$ did little to the amplitude of KCNQ current, its suppression by Oxo-M, or the subsequent recovery (Fig. 3a). OAG also did not change the time course of deactivation of channels at $-60 \mathrm{mV}$ or of activation at $-20 \mathrm{mV}$ (Fig. 3b), and it did not change the steady-state voltage dependence of activation measured from tail currents at $-70 \mathrm{mV}$ (Fig. 3c). DOG $(10 \mu \mathrm{M})$ was similar to OAG except that there was a tendency for overrecovery of current after Oxo-M application (Fig. 3d), the activation at $-20 \mathrm{mV}$ was significantly accelerated ( $\tau=114 \mathrm{~ms}$ vs $313 \mathrm{~ms}$ ) (Fig. $3 \mathrm{e}$ ), and the voltage dependence of activation was slightly shifted towards more negative potentials (Fig. 3f). Thus, this concentration of 
Fig. 4 a-f DAG probe translocation and current modulation with DAG kinase inhibitors. a, $\mathbf{d}$ Inhibitors of DAG kinase slow translocation of C1-EGFP probe induced by muscarinic stimulation. Cells preincubated with $20 \mu \mathrm{M}$ of the DAG kinase inhibitors R59022 (a) or R59949 (d) for $1 \mathrm{~h}$ were stimulated with Oxo-M $(10 \mu \mathrm{M})$ and the cytoplasmic fluorescence of C1-EGFP probe was measured using confocal microscopy. b, e Voltage-dependence of tail currents at $-70 \mathrm{mV}$ in control and after incubation with DAG kinase inhibitors. $\mathbf{c}, \mathbf{f}$ Effect of DAG kinase inhibitors on the muscarinic modulation of KCNQ current. Cells were preincubated with R59022 $(20 \mu \mathrm{M})$ or R59949 $(20 \mu \mathrm{M})$ for an hour

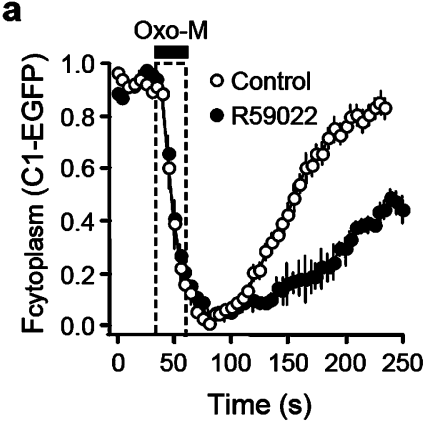

d

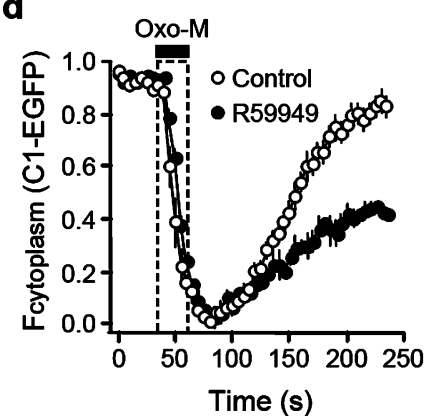

b

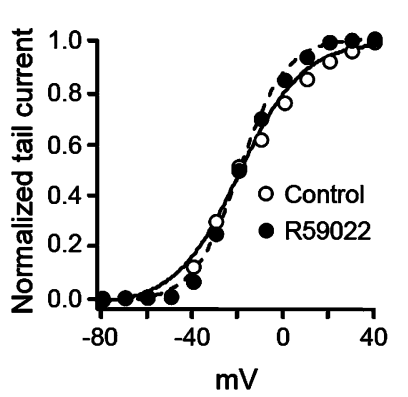

e

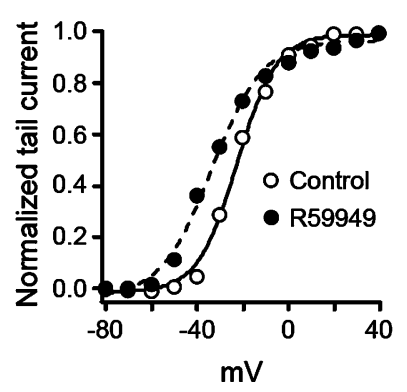

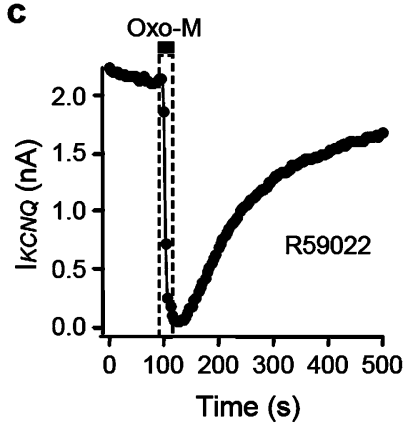

f

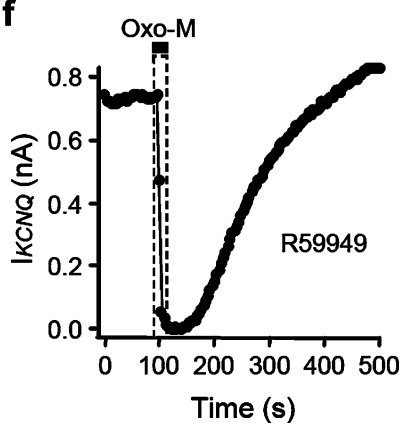

DAGs does not mimic or perturb the suppression and recovery of KCNQ current after short activation of PLC.

As some published works report effects of DAG at concentrations higher than $10 \mu \mathrm{M}$ DAG $[12,14,25]$, we also tested a tenfold-higher concentration using both OAG and DOG (data not shown). The effects with $100 \mu \mathrm{M}$ were similar to those with $10 \mu \mathrm{M}$ except that the KCNQ current was slightly depressed during DOG or OAG application (15.8 $\pm 0.7 \%, n=6$, and $6.2 \pm 1.0 \%, n=4$, respectively), an effect that took only a few seconds to develop and reverse. As before, DOG speeded activation of current and shifted the voltage dependence of activation (by $-8 \mathrm{mV}$ ). The higher concentration of DAG analogs also did not induce translocation of the $\mathrm{PIP}_{2}$ probe or change its translocation during Oxo-M application (data not shown), confirming the specificity of the probe and a lack of major change of PLC activation.

Pathways of DAG degradation

The preceding negative experiments could be criticized because they involved application of unnatural DAG analogs. Actions of these DAGs could be different, and certainly the downstream metabolites are not the same as those of native DAGs. The predominant endogenous DAG produced by PLC action on $\mathrm{PIP}_{2}$ would be 1-stearoyl-2arachidonyl-sn-glycerol [22]. One approach to testing the endogenous DAGs would be to slow their further metabolism so that they would persist longer and so downstream metabolites would not be produced. DAGs are metabolized in three ways by three major enzymes (Fig. 1a). DAG kinase phosphorylates DAG to give phosphatidic acid, which has potential signaling activity. DAG lipase and monoacylglycerol lipase remove the fatty chains, to give first monoacylglycerol and then glycerol plus the free fatty acids, stearic acid, and arachidonic acid, which have potential signaling roles. Finally, DAG acyltransferase can yield triglyceride, which is not expected to have signaling activity.

We began by using two inhibitors of DAG kinase. Figure $4 \mathrm{a}$,d shows that $20 \mu \mathrm{M}$ of the DAG kinase inhibitors R59022 and R59949 markedly slows the disappearance of DAG after activation of PLC, as assessed with confocal microscopy of our DAG translocation probe. Simulating these experiments with the kinetic model used for Fig. 1b allows us to estimate that the rate of DAG metabolism is slowed by a factor of 2 with these inhibitors, making the mean lifetime of DAG molecules about 160 s. Thus, we conclude that in our cells about $50 \%$ of the DAG is normally metabolized by DAG kinase to phosphatidic acid. The R59022 inhibitor had little effect by itself on the voltage dependence of activation of KCNQ channels (Fig. 4b), and the R59949 inhibitor caused a negative shift averaging $-13 \mathrm{mV}$ (Fig. 4e) and a speeding of the activation time constant at $-20 \mathrm{mV}(\tau=220 \pm 28,213 \pm 21$, and $94 \pm 9$ s for control, R59022, and R9949, respectively).

Despite doubling the lifetime of DAG and preventing formation of phosphatidic acid, these inhibitors do not reduce the depth of muscarinic suppression of current or slow the time course of recovery of channels from muscarinic suppression (Fig. 4c,f). The half-time for recovery of current was $155 \pm 8 \mathrm{~s}(n=5)$ in control cells 

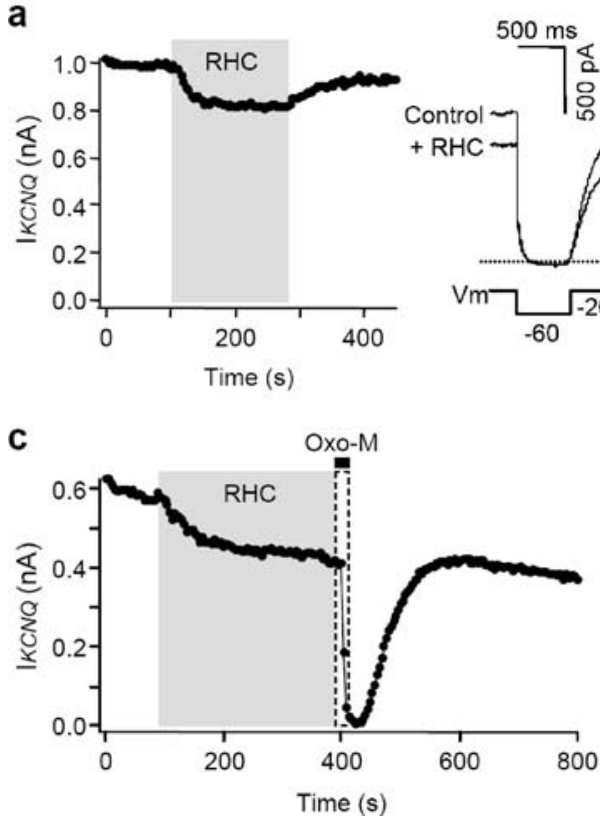

b
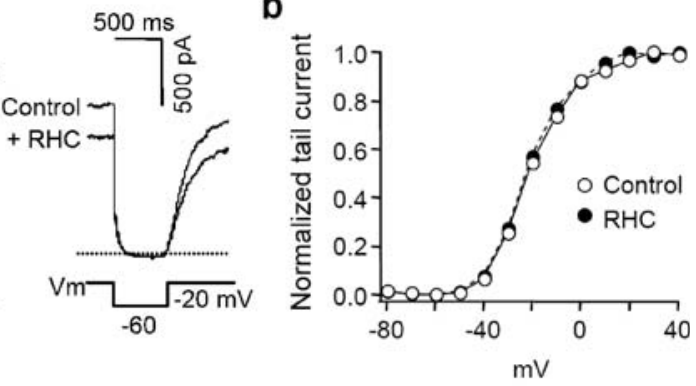

d

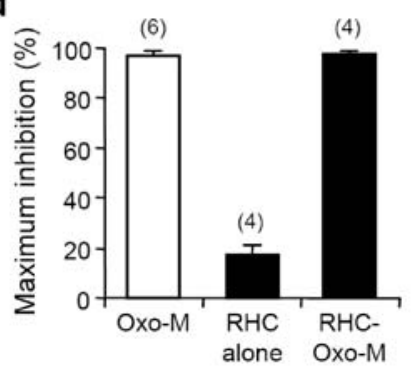

Fig. 5 a-d KCNQ current during application of DAG lipase inhibitor. a Reversible decrease of KCNQ current by the DAG lipase inhibitor RHC-80267 (RHC, $40 \mu \mathrm{M})$. Inset shows the waveforms before and after addition of RHC. b Voltage-dependence of tail currents at
$-70 \mathrm{mV}$ in control and after incubation with RHC for $5 \mathrm{~min}$. c Muscarinic modulation of KCNQ current after $40 \mu \mathrm{M}$ RHC. d Summary of relative current inhibition by Oxo-M or RHC

(Fig. 6a), and $500 \mathrm{nM}$ of the inactive 4- $\alpha$-phorbol myristate acetate (4- $\alpha$-PMA) did not translocate the probe (Fig. 6b). Nevertheless, although PMA effectively recruits the DAG probe (which contains the $\mathrm{C} 1$ domain of $\mathrm{PKC}$ ), neither PMA nor the control compound significantly changed the KCNQ current, its voltage dependence or its suppression and recovery following $10 \mu \mathrm{M}$ Oxo-M (Fig. 6c-f).

\section{Discussion}

We have looked for roles of DAG in the strong, acute modulation of KCNQ channels by activation of PLC, and we find none. Applications of exogenous OAG and DOG do not mimic or change this modulation. Inhibitors of endogenous DAG metabolism do not intensify or weaken muscarinic modulation and its recovery. Phorbol ester does not mimic or change the muscarinic suppression. Using a fluorescent translocation probe for DAG, we show that each of the reagents used is applied at a concentration that would be recognized by the $\mathrm{C} 1$ domain of $\mathrm{PKC}$ or that significantly prolongs the lifetime of endogenous DAG.

Our observations give two indices of DAG metabolism in tsA cells. We found that the mean lifetime of endogenous DAGs is $80 \mathrm{~s}$ at room temperature and that breakdown via DAG kinase to phosphatidic acid accounts for about $50 \%$ of the DAG metabolism. The 50\% figure assumes that the two DAG kinase inhibitors tested have high selectivity for this enzyme and inhibit it relatively completely. These 
Fig. 6 a-f Probe translocation and KCNQ current with phorbol ester. a, b Translocation of C1EGFP probe by active phorbol ester phorbol myristate acetate (PMA) (a, $500 \mathrm{nM})$ and not by inactive 4- $\alpha$-PMA (b, $500 \mathrm{nM}$ ). c, d Effect of PMA (c) or 4- $\alpha$ phorbol myristate acetate $(4-\alpha-$ PMA) (d) on the current and muscarinic modulation. e Voltage-dependence of tail currents at $-70 \mathrm{mV}$ in control and after PMA treatment. f Summary of the effect of phorbol esters on the amplitude of KCNQ current and the Oxo-M-induced current inhibition
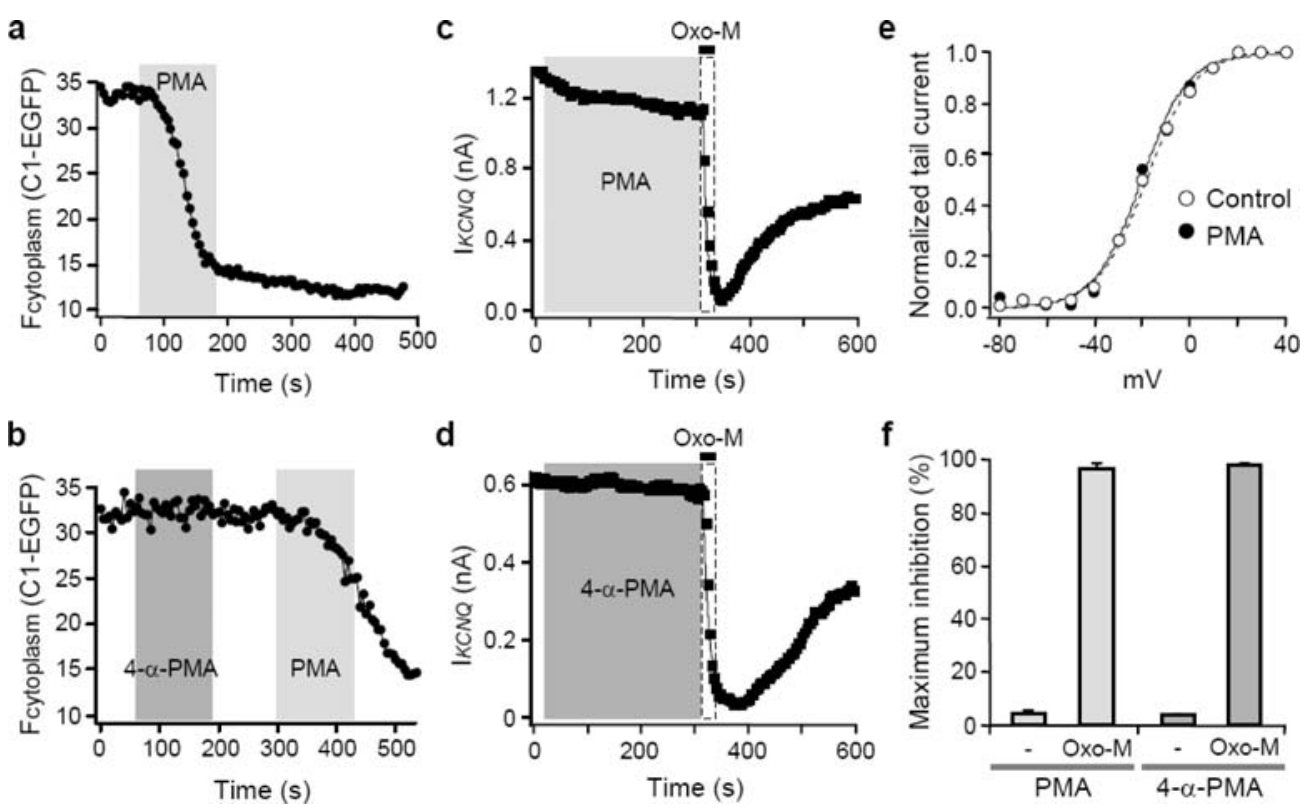

conclusions compare favorably with a study of DAG metabolism in platelets where the fate of added $\left[{ }^{3} \mathrm{H}\right] \mathrm{DOG}$ (labeled in the glycerol) was followed by chemical analysis of products [23]. The DOG was half metabolized in $60 \mathrm{~s}$ at $37^{\circ} \mathrm{C}$, and production of DOG phosphate by DAG kinase accounted for $40 \%$ of the loss. Cleavage to $\left[{ }^{3} \mathrm{H}\right]$ glycerol by di- and monoacylglycerol lipase accounted for another $20 \%$ of the loss. Eventually, $\left[{ }^{3} \mathrm{H}\right]$ dioctanolyphosphatidylinositol was also formed, presumably from the DOG phosphate (phosphatidic acid) product of DAG kinase.

Interestingly, this conversion had a half time of roughly $20 \mathrm{~min}$ at $37^{\circ} \mathrm{C}$, showing, as is usually stated, that the synthesis of $\mathrm{PIP}_{2}$ after PLC activation must depend much more on the existing large, unphosphorylated phosphatidylinositol pool than on slow regeneration all the way from phosphatidic acid. These experiments also remind us that during functional studies with exogenous DAG analogs, numerous metabolic steps are generating additional downstream products as well. Review articles (e.g., [22]) emphasize that much cellular DAG is formed from abundant lipids, such as phosphatidylcholine, by pathways that do not involve $\mathrm{PIP}_{2}$-specific PLC, as well as by dephosphorylation of phosphatidic acid. We have no chemical analyses but can say that the C1-probe, which recognizes a broad range of DAGs, does not report a lot of plasma membrane DAG accumulation in tsA cells until PLC is activated. Hence, the DAG pools in unstimulated cells seem small by this criterion.

It is not surprising that DAGs have no direct effect on KCNQ channel modulation, as there have been few prior suggestions that they might. But now it seems very clear. We can say this both for exogenous OAG and DAG, as well as for the endogenous DAG. We did find that DOG, but not OAG, seemed to have direct effects on channel gating, facilitating opening. The rate of activation upon depolarization to $-20 \mathrm{mV}$ was speeded and the voltage dependence of activation was shifted in such a way as to increase the probability of opening at any voltage. A similar facilitation of opening was seen with R59949, and not R59022. Further structure-activity studies would be needed to interpret these perturbations and to relate them to any physiological signals.

From our inhibitor work, we would argue that the downstream products of DAG metabolism are not needed for the acute muscarinic suppression of KCNQ current and its subsequent recovery. First, inhibiting the formation of phosphatidic acid does not bother suppression or recovery. A similar result with R59022 was obtained in bullfrog [24]. Second, all products of DAG metabolism (such as arachidonic acid) must be produced slowly, as DAG is normally metabolized with a time constant of $80 \mathrm{~s}$. This would mean that these products are not generated significantly during the onset of inhibition $(<7 \mathrm{~s})$ and start to accumulate only during the recovery phase for short agonist exposures. Our experiments do not test whether downstream products may have direct actions. For that, they should be tested individually. Indeed, there already is much literature saying that arachidonic acid application increases KCNQ currents (reviewed in [4]). Our point is that such actions would have to be slow to develop after application of a stimulus for PLC. Ford et al. [24] did find that if DAG kinase was inhibited with R59022, repeated PLC activation eventually slowed recovery of $\mathrm{M}$ current, presumably reflecting the stoppage of phosphatidylinositol regeneration from phosphatidic acid. 
The possibility of roles for PKC remains a more difficult question. From work on frog neurons and frog smooth muscle, several investigators proposed that PKC might mediate muscarinic suppression of $\mathrm{M}$ current [12-14]. A strong reduction of current by OAG and PMA in C. elegans KCNQ homologs (KQT channels) expressed in Xenopus has also been attributed to activation of PKC, and, of the mammalian channels, KCNQ5 was shown to be strongly affected by PKC and the others not [25]. Our early work on frog neurons showed that exogenous DAGs did reduce $\mathrm{M}$ current somewhat, an effect blocked by blockers of PKC [11]. We also showed that the PKC blockers did not block the acute muscarinic suppression of $\mathrm{M}$ current (see also [26]).

Hence, we concluded then that activation of PKC was not an essential link in muscarinic suppression. In this paper, we saw smaller effects of DAGs on expressed mammalian KCNQ2/KCNQ3 channels than we saw earlier on $\mathrm{M}$ current in frog neurons, in accordance with [25]. Recent molecular work with mammalian channels showed that mammalian KCNQ channels bind the A-kinase anchoring protein AKAP 150, which in turn can bind PKC [15]. Furthermore, it was found that activation of PKC can increase the sensitivity of KCNQ channels to muscarinic modulation, whereas in frog neurons the agonist sensitivity was said to be reduced by PKC [12].

Significant sensitization to muscarinic agonists could well be happening in our experiments but would not be apparent, as we used only supra-maximal concentrations of Oxo-M to evoke the suppression of current. Another possible reason we did not see larger effects of OAG, DOG, or PMA is the suggestion that in some unstimulated mammalian cell lines, the relevant phosphorylation of the channels is already complete [27]. In either case, we continue to hold that acute activation of PKC during muscarinic signaling is not necessary for the rapid muscarinic suppression of current that we are studying.

Acknowledgements We thank Greg Martin for help in the Keck Imaging Center and Lea Miller for expert technical assistance. This work was supported by National Institutes of Health grant NS08174.

\section{References}

1. Prestwich GD (2005) Visualization and perturbation of phosphoinositide and phospholipid signaling. Prostaglandins Other Lipid Mediat 77:168-178

2. Hilgemann DW, Feng S, Nasuhoglu C (2001) The complex and intriguing lives of $\mathrm{PIP}_{2}$ with ion channels and transporters. Sci STKE 2001:RE19

3. Suh BC, Hille B (2005) Regulation of ion channels by phosphatidylinositol 4,5-bisphosphate. Curr Opin Neurobiol 15:370-378

4. Delmas P, Brown DA (2005) Pathways modulating neural KCNQ/ M (Kv7) potassium channels. Nat Rev Neurosci 6:850-862
5. Horowitz LF, Hirdes W, Suh BC, Hilgemann DW, Mackie K, Hille B (2005) Phospholipase C in living cells: activation, inhibition, $\mathrm{Ca}^{2+}$ requirement, and regulation of $\mathrm{M}$ current. J Gen Physiol 26:243-262

6. Li Y, Gamper N, Hilgemann DW, Shapiro MS (2005) Regulation of Kv7 (KCNQ) $\mathrm{K}^{+}$channel open probability by phosphatidylinositol 4,5-bisphosphate. J Neurosci 25:9825-9835

7. Suh BC, Hille B (2002) Recovery from muscarinic modulation of $\mathrm{M}$ current channels requires phosphatidylinositol 4,5-bisphosphate synthesis. Neuron 35:507-520

8. Suh BC, Horowitz LF, Hirdes W, Mackie K, Hille B (2004) Regulation of KCNQ2/KCNQ3 current by G protein cycling: the kinetics of receptor-mediated signaling by Gq. J Gen Physiol 123:663-683

9. Winks JS, Hughes S, Filippov AK, Tatulian L, Abogadie FC, Brown DA, Marsh SJ (2005) Relationship between membrane phosphatidylinositol-4,5-bisphosphate and receptor-mediated inhibition of native neuronal M channels. J Neurosci 25:3400-3413

10. Zhang H, Craciun LC, Mirshahi T, Rohacs T, Lopes CM, Jin T, Logothetis DE (2003) $\mathrm{PIP}_{2}$ activates KCNQ channels, and its hydrolysis underlies receptor-mediated inhibition of $\mathrm{M}$ currents. Neuron 37:963-975

11. Bosma MM, Hille B (1989) Protein kinase C is not necessary for peptide-induced suppression of $\mathrm{M}$ current or for desensitization of the peptide receptors. Proc Natl Acad Sci U S A 86:2943-2947

12. Brown DA, Adams PR (1987) Effects of phorbol dibutyrate on M currents and $\mathrm{M}$ current inhibition in bullfrog sympathetic neurons. Cell Mol Neurobiol 7:255-269

13. Brown DA, Marrion NV, Smart TG (1989) On the transduction mechanism for muscarine-induced inhibition of M-current in cultured rat sympathetic neurones. J Physiol 413:469-488

14. Clapp LH, Sims SM, Singer JJ, Walsh JV Jr (1992) Role for diacylglycerol in mediating the actions of ACh on M-current in gastric smooth muscle cells. Am J Physiol 263:C1274-C1281

15. Hoshi N, Zhang JS, Omaki M, Takeuchi T, Yokoyama S, Wanaverbecq N, Langeberg LK, Yoneda Y, Scott JD, Brown DA, Higashida H (2003) AKAP150 signaling complex promotes suppression of the M-current by muscarinic agonists. Nat Neurosci 6:564-571

16. Andoh $\mathrm{T}$, Itoh $\mathrm{H}$, Higashi $\mathrm{T}$, Saito $\mathrm{Y}$, Ishiwa $\mathrm{D}$, Kamiya $\mathrm{Y}$, Yamada Y (2004) PKC-independent inhibition of neuronal nicotinic acetylcholine receptors by diacylglycerol. Brain Res 1013:125-133

17. Dietrich A, Kalwa H, Rost BR, Gudermann T (2005) The diacylgylcerol-sensitive TRPC3/6/7 subfamily of cation channels: functional characterization and physiological relevance. Pflugers Arch 451:72-80

18. Hardie RC (2003) TRP channels in Drosophila photoreceptors: the lipid connection. Cell Calcium 33:385-393

19. Varnai P, Balla T (1998) Visualization of phosphoinositides that bind pleckstrin homology domains: calcium- and agonist-induced dynamic changes and relationship to myo- $\left[{ }^{3} \mathrm{H}\right]$ inositol-labeled phosphoinositide pools. J Cell Biol 143:501-510

20. Oancea E, Teruel MN, Quest AF, Meyer T (1998) Green fluorescent protein (GFP)-tagged cysteine-rich domains from protein kinase $\mathrm{C}$ as fluorescent indicators for diacylglycerol signaling in living cells. J Cell Biol 140:485-498

21. Stauffer TP, Ahn S, Meyer T (1998) Receptor-induced transient reduction in plasma membrane $\operatorname{PtdIns}(4,5) \mathrm{P}_{2}$ concentration monitored in living cells. Curr Biol 8:343-346

22. Wakelam MJ (1998) Diacylglycerol-when is it an intracellular messenger? Biochim Biophys Acta 1436:117-126

23. Bishop WR, Bell RM (1986) Attenuation of $s n$-1,2-diacylglycerol second messengers. Metabolism of exogenous diacylglycerols by human platelets. J Biol Chem 261:12513-12519 
24. Ford CP, Stemkowski PL, Light PE, Smith PA (2004) Experiments to test the role of phosphatidylinositol 4,5-bisphosphate in neurotransmitter-induced M-channel closure in bullfrog sympathetic neurons. J Neurosci 23:4931-4941

25. Wei AD, Butler A, Salkoff L (2005) KCNQ-like potassium channels in Caenorhabditis elegans. Conserved properties and modulation. J Biol Chem 280:21337-21345
26. Stemkowski PL, Tse FW, Peuckmann V, Ford CP, Colmers WF, Smith PA (2002) ATP-inhibition of M current in frog sympathetic neurons involves phospholipase $\mathrm{C}$ but not $\mathrm{InsP}_{3}, \mathrm{Ca}^{2+}$, PKC, or Ras. J Neurophysiol 88:277-288

27. Nakajo K, Kubo Y (2005) Protein kinase C shifts the voltage dependence of $\mathrm{KCNQ} / \mathrm{M}$ channels expressed in Xenopus oocytes. J Physiol 569:59-74 